\title{
Combination of Single Tuned Filter and Double Tuned Filter to Reducing Current Harmonics
}

\author{
Azmi Rizki Lubis ${ }^{1}$, Marwan Affandi², Mustamam ${ }^{3}$ \\ \{azmirizkilubis@unimed.ac.id ${ }^{1}$, mr1.affandi@gmail.com ${ }^{2}$ \} \\ Universitas Negeri Medan, Indonesia ${ }^{1,2,3}$
}

\begin{abstract}
This paper presents the results of the analysis of a combination of a single tuned filter (STF) and a double tuned filter (DTF) to reducing harmonic currents in a three-phase system. From the research that has been done, obtained significant results in reducing the current harmonic content in the three-phase system. THDi from $25.49 \%$ reduced to $14.84 \%$ and the $5^{\text {th }}$ harmonic content is $21.69 \%$ reduced to $13.45 \%$, the $7^{\text {th }}$ harmonic content is $10.26 \%$ reduced to $4.63 \%$, the $11^{\text {th }}$ harmonic content is $6.42 \%$ reduced to $2.33 \%$, and the $13^{\text {th }}$ harmonic content is $4.46 \% \%$ reduced to $1.32 \%$.
\end{abstract}

Keywords: Harmonic, Single Tuned Filter, Double Tuned Filter.

\section{Introduction}

The quality of electric power lately has been very worrisome which will affect electric power equipment [1]. Harmonic currents arise due to nonlinear loads such as power electronic equipment, UPS, arc furnaces, adjustable speed drives (ASD) so that the current waveform is no longer sinusoidal. Damaging effects have also been proven to be caused by the presence of harmonics such as overheating of transformer equipment, rotating machines, switchgear, bank capacitors. Induction motors also work at sub synchronous speeds and won't start cogging. The breaker equipment also does not work normally. Capacitors also fail to work due to increase dielectric pressure and excessive heat. The condition of the safety relay will also become more erratic and also the current regulation over time on the fuse may also change [2]. To reduce harmonics one of the cheapest and most economical ways is to use passive filters. The use of passive filters has also been widely used in high voltage direct current systems (HVDC) [3].

Single Tuned Filter (STF) is one of several types of passive filters used in reducing harmonics. STF consists of capacitors and reactors connected in series that is set to low harmonic frequencies. In the harmonics STF set is the value of the capacitor and reactor so that later the reactance value is the same, and the filter has a pure resistive value. Filter impedance is a capacitive value for low harmonics and inductive values for high harmonics, so that eventually the impedance value will get worse under the lowest regulated frequency [4]. To reduce two harmonics at once you can use Double Tuned Filter (DTF) because the scope of work area of DTF is wider than STF [5]. In this paper two passive filters, namely STF and DTF, will be combined to be designed to reduce the harmonic content in three-phase system. 


\section{Harmonic Distortion}

The condition of deviating voltage and current waves from sinusoidal waves at the ideal frequency of the electrical energy channel is the understanding of harmonics. Harmonics are also considered as the sum of different sine waves at different frequencies which are integral to the fundamental frequency. The sum ratio of all harmonic components and fundamental components is also called total harmonic distortion (THD). Equipment that is widely used in industries such as inverters, converters, cyclo-converters, battery charging, uninterruptible power supply (UPS). In electric power distribution system, if it supplies a nonlinear load, it will cause harmonic so there will be a mixture of voltage and fundamental current with harmonic voltage and current, eventually forming distortion [6].

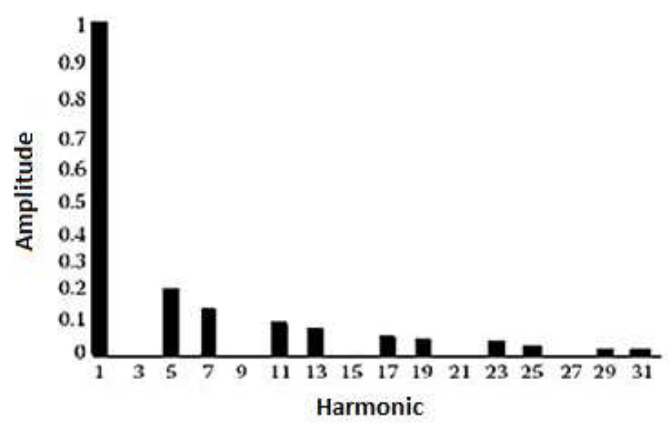

Fig. 1. Harmonic Spectrum

With the existence of harmonic currents, the cable in the distribution channel will be hot, an error in breaking the circuit breaker and also produce excessive heat in the transformer so that the life of the transformer will also be reduced [7].

\section{Harmonic Passive Filter}

The use of passive filters can reduce harmonics efficiently, where basically the principle of this passive filter is to regulate the components of the reactor and inductor at the frequency to be reduced harmonic content. The impedance of the filter is set low so that the harmonics will flow through the filter that has been set [8]. According to the connection, the passive filter consists of two types, namely the series passive filter and shunt passive filter. Current at full load will flow in the series passive filter while some current at full load will flow at the shunt passive filter. The use of passive shunt filters also requires a low cost and also reactive power at fundamental frequencies can also be flowed, therefore passive shunt filters are good for harmonic filters.

\section{Single Tuned Filter}

In the filter industry the most widely used to overcome harmonics in electric power systems is the Single Tuned Filter (STF). The resonant frequency at STF has a small impedance so that the current that has a value equal to the resonant frequency will be flowed through the filter so it does not flow into the system. The components contained in the STF are resistance $(\mathrm{R})$, inductance (L), and capacitance (C) connected in series with configurations as in Fig. 2. following. 


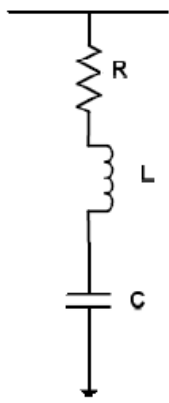

Fig. 2. Single Tuned Filter Configuration

To design STF the first thing to do is to determine the right capacitor value so that it gets a good power factor at fundamental frequencies. Previously, the capacitor reactance value $\left(X_{C}\right)$ and the capacitor reactive power must be determined as in the following equation,

$$
X_{C}=\frac{V^{2}}{Q_{C}}
$$

Where $V^{2}$ is the line to line voltage of the capacitor, $Q_{C}$ is the reactive power of the capacitor. After the $X_{C}$ value is obtained, the capacitance of the capacitor can be calculated using the following equation,

$$
C=\frac{1}{2 \pi f_{0} X_{C}}
$$

Where $f_{0}$ is the fundamental frequency. After the capacitor value is obtained, the next step is to find the reactor value of the filter which was previously searched for the inductive reactance value of the $X_{L}$ reactor using the following equation,

$$
X_{L}=\frac{X_{C}}{h_{n}^{2}}
$$

Where $h_{n}^{2}$ is the harmonic $\mathrm{n}_{\mathrm{th}}$. After the $X_{L}$ value is obtained, the reactor value can be found by using the equation,

$$
L=\frac{1}{2 \pi f_{0}}
$$

The last step is to determine the value of the resistance $(\mathrm{R})$ filter, to get the resistance value (R) filter first to find the characteristic reactance of the filter $\left(X_{n}\right)$ by using the equation,

$$
X_{n}=h_{n} X_{L}
$$

After obtaining the characteristic reactance value of the filter, the resistance $(R)$ of the filter can be found a by using the following equation.

$$
R=\frac{X_{n}}{Q}
$$




\section{Double Tuned Filter}

The simplest use of DTF has almost pure inductive impedance at all frequencies. Resonance will be generated if the impedance of the system is approximately equal and opposite to the filter at several harmonic frequencies. The resonance generated in time will cause large harmonic currents coupled with excessive voltage harmonics from filter components and in the system. So that this does not happen it is necessary to add a dampening resistor in a different way so that some damped DTF configurations can be shown in Fig. 3.

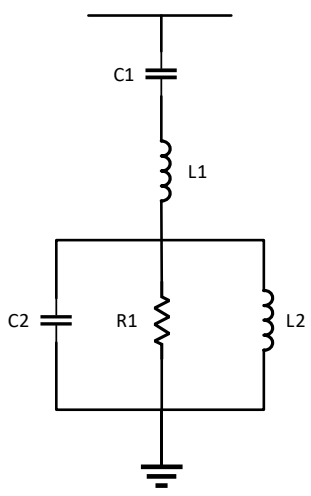

(a)

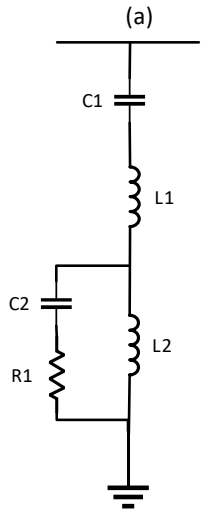

(d)

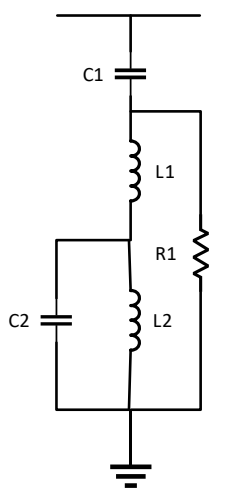

(b)

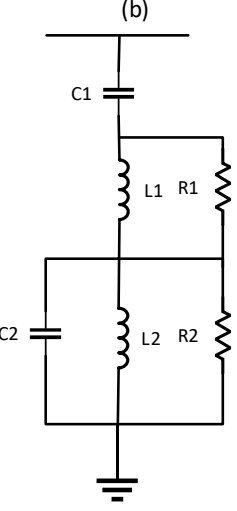

(e)
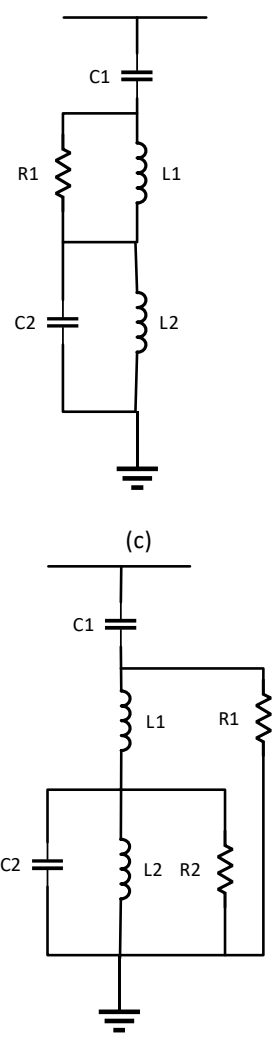

(f)

Fig. 3. Configurations of damped-type double tuned filter

Of the several types of DTF in Fig. 3 most used are in Fig. 3 (a). Conventional DTF is a series resonant circuit consisting of two inductors and two capacitors as in Fig. 1. 


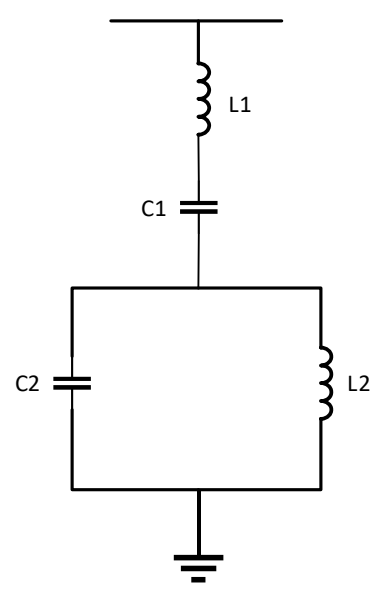

Fig. 4. Double Tuned Filter Conventional

From DTF on Fig. 4 can be searched for series resonance frequency and parallel resonance frequency using the following equation,

$$
\left[\begin{array}{l}
\omega_{s} \\
\omega_{p}
\end{array}\right]=\left[\begin{array}{c}
\frac{1}{\sqrt{L_{1} C_{1}}} \\
\frac{1}{\sqrt{L_{2} C_{2}}}
\end{array}\right]
$$

To find the large DTF impedance in Fig. 2 then you can use the following equation,

$$
\begin{aligned}
Z_{f} & =j \omega L_{1}+\frac{1}{j \omega C_{1}}+\left(j \omega C_{2}-j \frac{1}{\omega L_{2}}\right)^{-1} \\
& =\frac{\left(1-\frac{\omega^{2}}{\omega_{s}^{2}}\right)\left(1-\frac{\omega^{2}}{\omega_{p}^{2}}\right)-\omega^{2} L_{2} C_{1}}{j \omega C_{1}\left(1-\frac{\omega^{2}}{\omega_{p}^{2}}\right)}
\end{aligned}
$$

It takes the calculation of the parameter $C_{1}$ to analyze the coefficient of $\omega$ using the following equation,

$$
C_{1}=C_{a}+C_{b}
$$

Next find the value of $L_{1}$ on DTF by using the following equation,

$$
L_{1}=\frac{1}{C_{a} \omega_{a}^{2}+C_{b} \omega_{b}^{2}}
$$

Then find the value of $L_{2}$ on DTF by using the following equation,

$$
L_{2}=\frac{\left(1-\frac{\omega_{a}^{2}}{\omega_{s}^{2}}\right)\left(1-\frac{\omega_{b}^{2}}{\omega_{p}^{2}}\right)}{C_{1} \omega_{a}^{2}}
$$


Next find the value of $C_{2}$ can use the following equation,

$$
C_{2}=\frac{1}{L_{2} \omega_{p}^{2}}
$$

Where to get the value of $\omega_{1}$ and $\omega_{2}$ using the following equation,

$$
\begin{aligned}
& \omega_{s}=\frac{1}{\sqrt{L_{1} C_{1}}} \\
& \omega_{p}=\frac{\omega_{a} \omega_{b}}{\omega_{S}}
\end{aligned}
$$

\section{Experimental Results}

This paper presents the reduction of current harmonic content in a three-phase system with a voltage of 220 volts with a frequency of $50 \mathrm{~Hz}$ which will be installed in a combination of STF and DTF. There are two STF used to reduce harmonic currents $5^{\text {th }}$ and harmonic currents $7^{\text {th }}$, while DTF is used to reduce harmonic currents $11^{\text {th }}$ and harmonic currents $13^{\text {th }}$. Fig. 6 shows modeling of the three-phase system after the installation of STF and DTF, Fig. 7 shows the current wave before filter installation and Fig. 8 shows the current waveform after the installation of STF and DTF.

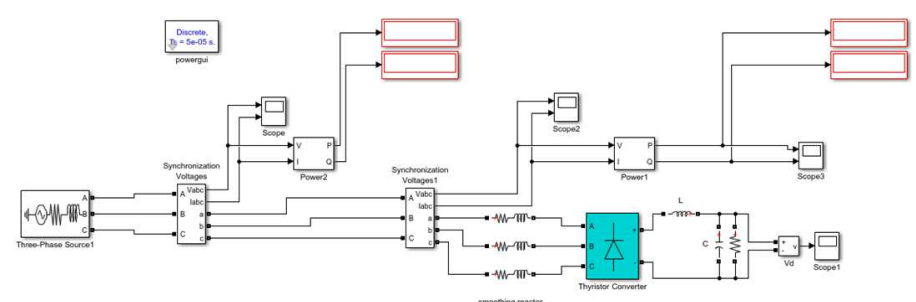

Fig. 5. Modeling three-phase systems before filter installation

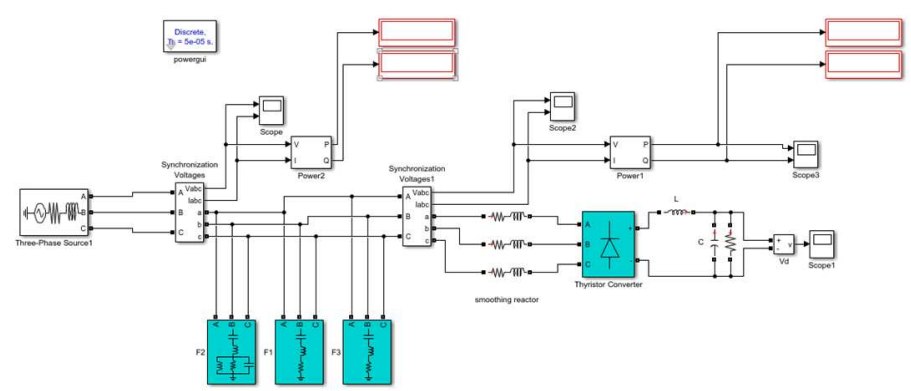

Fig. 6. Modeling three-phase systems after filter installation 


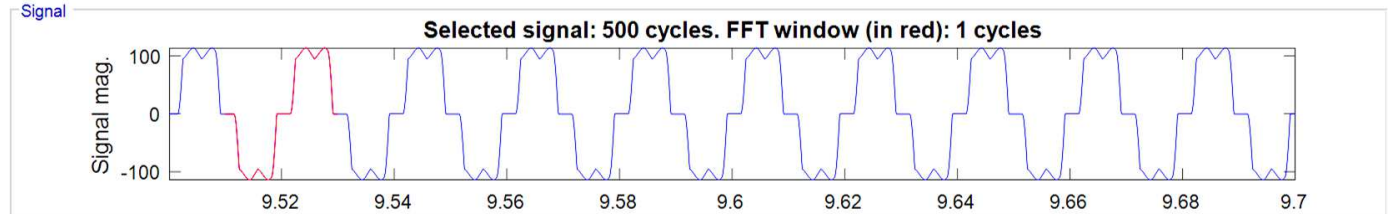

Fig. 7. Current wave before filter installation

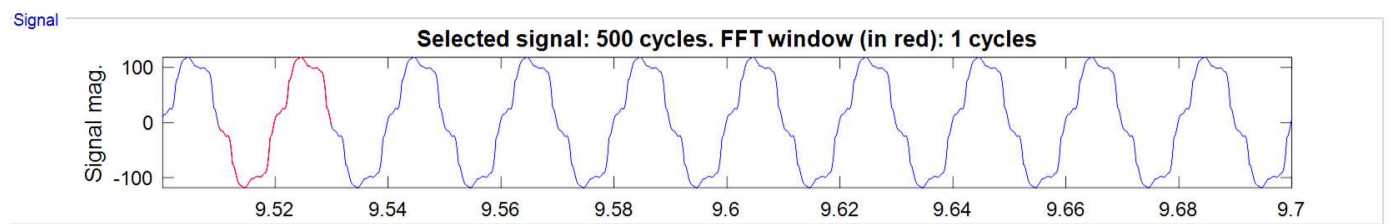

Fig. 8. Current wave after filter installation

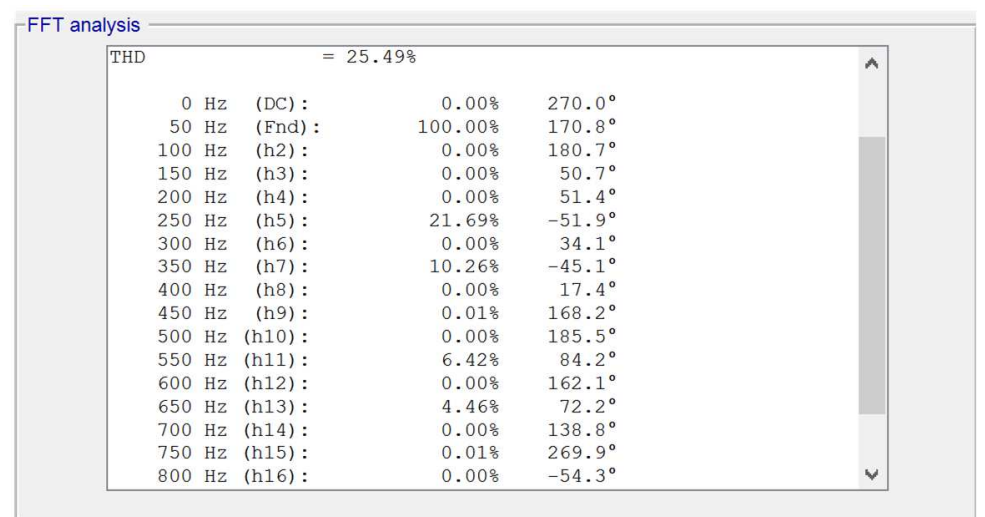

Fig. 9. Current Harmonic before filter installation

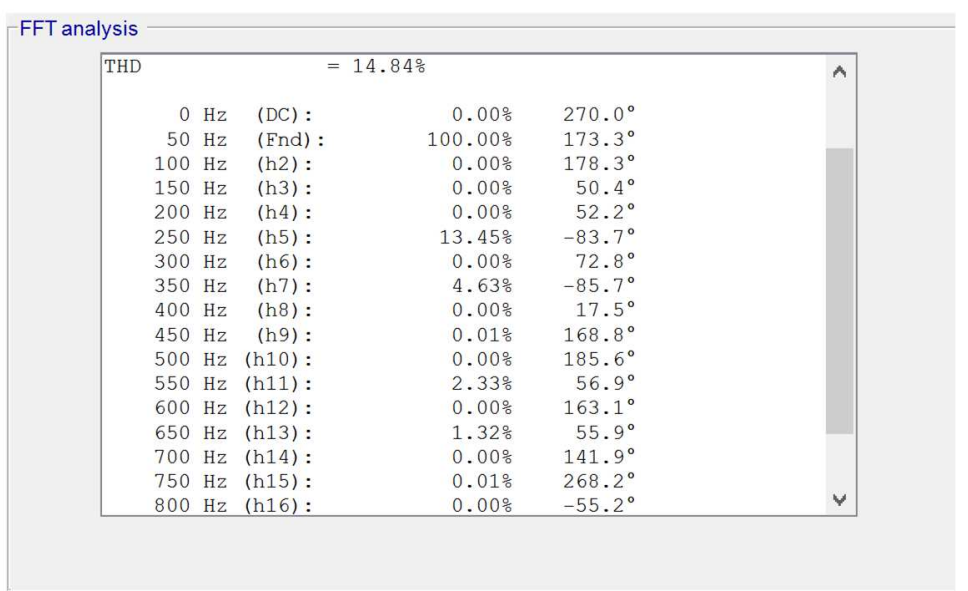

Fig. 10. Current Harmonic after filter installation 


\section{Conclusion}

The results obtained from the study by combining STF and DTF in a three-phase nonlinear load system have succeeded in reducing the harmonic content caused by non-linear loads. The combination of STF and DTF has succeeded in reducing current THD from $25.49 \%$ to $14.84 \%$, and the 5 th harmonic content was $21.69 \%$ reduced to $13.45 \%$, the 7 th harmonic was $10.26 \%$ reduced to $4.63 \%$, the 11 th harmonic was $6.42 \%$ reduced to $2.33 \%$, and the 13 th harmonic by $4.46 \%$ reduced to $1.32 \%$.

\section{References}

[1] A. Baitha, A Comparative Analysis of Passive Filters for Power Quality Improvement pp. 327-332, (2015).

[2] J. C. Mccall, Design of Filters to Reduce Harmonic Distortion Industrial Power Systems in, vol. I, no. 3, pp. 504-511, (1987).

[3] S. N. A. L. Yousif, M. Z. C. Wanik, I. A. Mohamed, and S. M. Ieee, Implementation of Different Passive Filter Designs for Harmonic Mitigation, (2004).

[4] G. J. Wakileh, Power Systems Harmonics: Fundamentals, Analysis and Filter Design. Springer, (2001).

[5] X. Yao, Algorithm for the parameters of double tuned filter, in 8th International Conference on Harmonics and Quality of Power. Proceedings (Cat. No.98EX227), vol. 1, pp. 154-157 vol.1, (1998).

[6] J. P. Nelson, A better understanding of harmonic distortion in the petrochemical industry, IEEE Trans. Ind. Appl., vol. 40, no. 1, pp. 220-231, (2004).

[7] R. D. Henderson and P. J. Rose, Harmonics: the effects on power quality and transformers, IEEE Trans. Ind. Appl., vol. 30, no. 3, pp. 528-532, (1994).

[8] D. D. Reljić, V. V Vasić, and D. V Oros, Power factor correction and harmonics mitigation based on phase shifting approach, in 2012 15th International Power Electronics and Motion Control Conference (EPE/PEMC), p. DS3b.12-1-DS3b.12-8, (2012). 